\title{
DISSECTION OF THE FUSION MACHINE OF SARS-CORONAVIRUS
}

\author{
Megan W. Howard, Brian Tripet, Michael G. Jobling, Randall K. Holmes, \\ Kathryn V. Holmes, and Robert S. Hodges*
}

\section{INTRODUCTION}

Infection of target cells by enveloped viruses occurs through two pathways. Upon binding to a receptor on the cell surface, the virus enters the cell either through the plasma membrane or via endocytosis through a $\mathrm{pH}$-dependent fusion event with the endosomal membrane. ${ }^{1}$ Coronaviruses (CoVs) enter host cells using both pathways ${ }^{1,2}$ through the interaction of the $\mathrm{S}$ (spike) glycoprotein with the target receptor on the cell surface. Different CoVs use different receptors to bind to cells and enter either at the plasma membrane or via endosomes. Previous work from our lab has identified murine CEACAM1a as a receptor for MHV. ${ }^{4}$ SDS-PAGE and liposome flotation analysis showed that either soluble receptor or basic $\mathrm{pH}(\mathrm{pH} 8.0)$ at $37^{\circ} \mathrm{C}$ triggers the $\mathrm{S}$ protein to undergo a conformational change that exposes trypsin cleavage sites and a hydrophobic region of the protein that can associate with membranes. ${ }^{3}$

The S protein of CoVs is a type-I viral fusion glycoprotein (VFG), ${ }^{5}$ related to the fusion glycoproteins of many other enveloped viruses (including gp41 of HIV-1, gp2 of Ebola, HA of Influenza, and F of SV5)., 7 Type-I VFGs contain several conserved domains; two heptad repeats (HR1 and HR2 toward the N- and C-termini of the fusion domain of the protein, respectively), a hydrophobic fusion peptide (FP), and a transmembrane domain (TM). Some of these proteins also contain a membrane-proximal domain (TMP) rich in aromatic amino acids. ${ }^{7}$

Binding of the $\mathrm{S}$ protein of $\mathrm{CoVs}$ to a specific receptor induces conformational changes in the $\mathrm{S}$ protein, changing it from an inactive pre-fusion state to a fusion-active state. This allows a previously hidden hydrophobic region of the protein, the fusion peptide, to interact with the host membrane. Another conformational change occurs that changes the trimeric $\mathrm{S}$ protein into a post-fusion conformation, containing a structure known as a 6-helix-bundle $(6 \mathrm{HB})$ which draws the host and viral membranes close together, facilitating membrane fusion and entry. The $6 \mathrm{HB}$ is formed through the

*University of Colorado Health Sciences Center, Aurora, Colorado 80045. 
antiparallel association of the C-terminal HR with the N-terminal HRs in a 'trimer of dimers' conformation. ${ }^{7}$

Although CoV S proteins are type-I VFGs, they have several unusual features. Most VFGs must be cleaved by a serine protease before the protein is able to mediate membrane fusion. However some CoVs (e.g., HCoV-229E) function without a known serine protease activation event. The $\mathrm{FP}$ of $\mathrm{CoV} \mathrm{S}$ proteins is not at the $\mathrm{N}$ terminus of the fusion domain, as seen with most type-I VFGs. Instead, the CoV FP is internal, preceded by $\sim 230$ aa's. A long interhelical domain of $\sim 140$ aa's links the two HRs. Thus S proteins of CoVs are variant type-I VFGs.

\section{RESULTS AND DISCUSSION}

To investigate the SARS-CoV S protein and its mechanism of fusion, we used bioinformatics to identify regions likely to be involved in membrane fusion. Using STABLECOIL, ${ }^{8}$ a program based on empirical data on coiled coils, we identified portions of the S protein likely to fold into coiled coils. We identified two regions, both in the S2 domain: a N-terminal (HRN, aa882-1011), and a C-terminal (HRC, aa1151-1185) region, separated by a domain of $\sim 140$ aa residues (Fig 1). ${ }^{9}$ The HRN region could be subdivided into three parts based on the heptad $a$ and $d$ positions. The presence of these alternative heptad 'registers' may indicate that the HRN region can fold into two structurally different coiled coils. Two of these 'registers' overlap, indicating that hydrophobic residues are present both in the interfacial $e$ and $g$ positions and in the intrafacial $a$ and $d$ positions. This 'double register' may be involved in HRC binding and association with HRN in the post-fusion state.

We synthesized different regions of the HRN and HRC regions in overlapping 35-aa peptides. Analysis of these peptides for helical structure and stability using circular dichroism (CD) spectroscopy and temperature denaturation curves showed that the HRN peptides were random coil. However addition of 50\% trifluoreoethanol (TFE), a helixinducing solvent, showed that they could adopt a helical structure, thus they do have intrinsic helical character. The HRC peptide showed helical structure, however it was of low helicity and stability $\left(50 \%\right.$ unfolded at $\left.33^{\circ} \mathrm{C}\right)$. Mixing $\mathrm{HRN}$ and HRC peptides showed that the HRN10 peptide, corresponding to aa 916-950 of SARS S, could interact with the HRC peptide as the stability of the complex rose $24^{\circ}$ to $57^{\circ} \mathrm{C}$ indicating a stabilizing interaction. The $\mathrm{CD}$ spectra of the complex indicated an alpha-helical structure. We examined the interaction of the HRN10 peptide with truncated HRC peptides. The temperature denaturation curves were similar for the full HRC and the truncated HRC4 (aa 1151-1185), indicating that the binding region of the HRN10 peptide (aa 916-950) on HRC is localized to aa 1151-1185.

Sedimentation equilibrium (SE) was used to determine the oligomeric states of the HRN (aa 882-973) and HRC peptides individually. The SE data indicated that HRC, aa 1151-1185, associates as a trimer. Surprisingly the HRN peptide associated in a tetrameric state. These results suggest that though the HRN is a trimer in the complete protein, truncation of the native sequence causes it to adopt a tetrameric configuration. The SE data on the HRN10/HRC complex fits best to a model with a 3:3 molar ratio of HRN10:HRC, indicating formation of a $6 \mathrm{HB}$. 


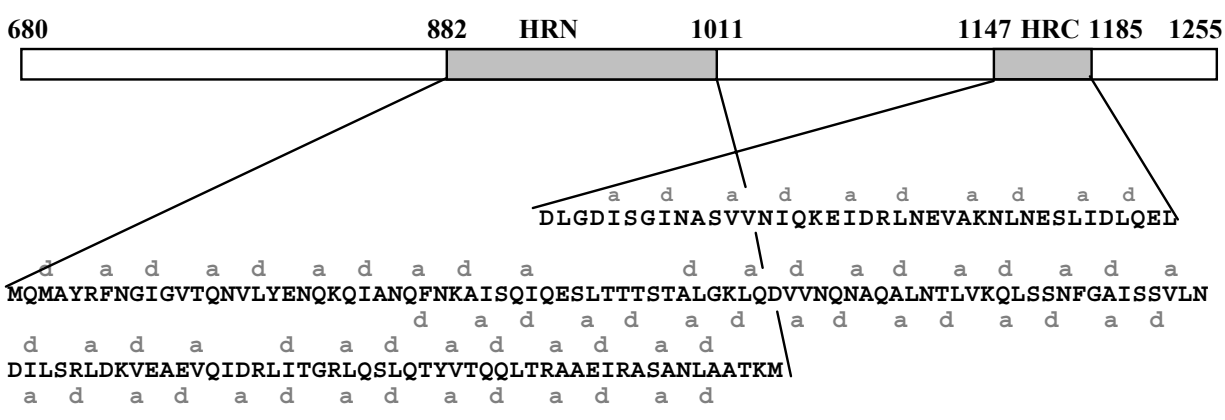

Figure 1. SARS-CoV S2 domain.

We investigated the orientation of the helicies in the HRN10/HRC complex by introducing disulfide bridges. Engineering a gly-gly-cys sequence onto the N- or Ctermini of HRC and the N-termini of the HRN10 peptide allowed us to covalently link the peptides together, locking them into either a highly stable soluble form (the correct orientation) or a less stable form composed of head-to-tail aggregates (the incorrect orientation). Temperature denaturation studies showed that the antiparallel orientation was favored by an increase in stability (by $19^{\circ} \mathrm{C}$ ) and solubility $(>5 \mathrm{mg} / \mathrm{mL})$. Surprisingly, the CD spectra of the covalently linked antiparallel complex $\left(-19200^{\circ} * \mathrm{~cm}^{2} * \mathrm{dmol}^{-1}\right.$ at 222 $\mathrm{nm}$ rather than $\left.-34,500^{\circ} * \mathrm{~cm}^{2} * \mathrm{dmol}^{-1}\right)$ showed that it either contained unfolded residues at one end, or one of the peptides exists in a partially helical form. The crystal structure of the $6 \mathrm{HB}$ of SARS S was recently published, and confirmed that the $6 \mathrm{HB}$ is not $100 \%$ helical and contains extended regions at both ends of HRC. ${ }^{11,15}$

\section{CONCLUSIONS}

We characterized the HRN and HRC regions in the ectodomain of the SARS-CoV S protein, a variant type-I VFG, and identified similarities to the MHV fusion core ${ }^{16}$ and other type-I VFG fusion cores. ${ }^{6,7}$ Analysis of the HRN region showed that the region that interacts with HRC is aa 916-950. Peptides of HRN, aa 916-950, and HRC, aa 11511185 , can form a $6 \mathrm{HB}$, which appears similar to the interactions between the HRC and HRN of the SV5 F protein ${ }^{6}$ that involves both helical and extended regions in HRC. The identification of the $6 \mathrm{HB}$ structure formed by the HRN and HRC peptides of the SARS$\mathrm{CoV}$ fusion core suggests that the HRC peptide may inhibit viral fusion and entry, as observed with similar VFGs. ${ }^{10-15}$ Recent publications show that the HRC peptide can inhibit infection with SARS-CoV. ${ }^{10-15}$

\section{ACKNOWLEDGMENTS}

We thank Mark Genest, Jennifer Labrecque, Larissa Thackray, and Mark Young for help with peptide synthesis, cloning, and purification. CD and analytical ultracentrifugation measurements were performed at the Biophysics Core Facility, University of Colorado Health Sciences Center. This work was supported by NIH grants RO1 A148717 (to 
R.S.H.), RO1 AI31940 (to R.K.H.), RO1 A125231 (to K.V.H.), and T32 AI52066 (for M.W.H.).

\section{REFERENCES}

1. L. J. Earp, S. E. Delos, H. E. Park, and J. M. White, The many mechanisms of viral membrane fusion proteins, Curr. Top. Microbiol. Immunol. 285, 25-66 (2004).

2. T. C. Nash and M. J. Buchmeier, Entry of mouse hepatitis virus into cells by endosomal and nonendosomal pathways, Virology 233, 1-8 (1997).

3. B. D. Zelus, J. H. Schickli, D. M. Blau, S. R Weiss, and K. V. Holmes, Conformational changes in the spike glycoprotein of murine coronavirus are induced at 37 degrees $\mathrm{C}$ either by soluble murine CEACAM1 receptors or by $\mathrm{pH} 8, J$. Virol. 77, 830-840 (2003).

4. G. S. Dveksler, C. W. Dieffenbach, C. B. Cardellichio, K. McCuaig, M. N. Pensiero, G. S. Jiang, N. Beauchemin, and K. V. Holmes, Several members of the mouse carcinoembryonic antigen-related glycoprotein family are functional receptors for the coronavirus mouse hepatitis virus-A59, J. Virol. 67, 1-8 (1993).

5. B. J. Bosch, R. van der Zee, C. A. de Haan, and P. J. Rottier, The coronavirus spike protein is a class I virus fusion protein: structural and functional characterization of the fusion core complex, J. Virol. 77, 8801-8811 (2003).

6. K. A. Baker, R. E. Dutch, R A. Lamb, and T. S. Jardetzky, Structural basis for paramyxovirus-mediated membrane fusion, Mol. Cell 3, 309-319 (1999).

7. P. M. Colman and M. C. Lawrence, The structural biology of type I viral membrane fusion, Nat. Rev. Mol. Cell Biol. 4, 309-319 (2003).

8. B. Tripet and R. S. Hodges, in Peptides: The Wave of the Future, edited by M. Lebl and R. A. Houghten (American Peptide Society, San Diego, 2001), pp. 365-366.

9. B. Tripet, M. W. Howard, M. Jobling, R. K. Holmes, K. V. Holmes, and R. S. Hodges, Structural characterization of the SARS-coronavirus spike S fusion protein core, J. Biol. Chem. 279, 20836-20849 (2004).

10. B. J. Bosch, B. E. Martina, R. van der Zee, J. Lepault, B. J. Haijema, C. Versluis, A. J. Heck, R. De Groot, A. D. Osterhaus, and P. J. Rottier, Severe acute respiratory syndrome coronavirus (SARS-CoV) infection inhibition using spike protein heptad repeat-derived peptides, Proc. Natl. Acad. Sci. USA 101, 8455-8460 (2004).

11. Y. Xu, Z. Lou, Y. Liu, H. Pang, P. Tien, G. F. Gao, and Z. Rao, Crystal structure of severe acute respiratory syndrome coronavirus spike protein fusion core, J. Biol. Chem. 279, 49414-49419 (2004).

12. S. Liu, G. Xiao, Y. Chen, Y. He, J. Niu, C. R. Escalante, H. Xiong, J. Farmar, A. K. Debnath, P. Tien, and S. Jiang, Interaction between heptad repeat 1 and 2 regions in spike protein of SARS-associated coronavirus: implications for virus fusogenic mechanism and identification of fusion inhibitors, Lancet $\mathbf{3 6 3}$, 938-947 (2004).

13. Y. Xu, J. Zhu, Y. Liu, Z. Lou, F. Yuan, Y. Liu, D. K. Cole, L. Ni, N. Su, L. Qin, X. Li, Z. Bai Z., J. I. Bell, H. Pang, P. Tien, G. F. Gao, and Z. Rao, Characterization of the heptad repeat regions, HR1 and HR2, and design of a fusion core structure model of the spike protein from severe acute respiratory syndrome (SARS) coronavirus, Biochemistry 43, 14064-14074 (2004).

14. K. Yuan, L. Yi, J. Chen, X. Qu, T. Qing, X. Rao, P. Jiang, J. Hu, Z. Xiong, Y. Nie, X. Shi, W. Wang, C. Ling, X. Yin, K. Fan, L. Lai, M. Ding, and H. Deng, Suppression of SARS-CoV entry by peptides corresponding to heptad regions on spike glycoprotein, Biochem. Biophys. Res. Commun. 319, 746-752 (2004).

15. P. Ingallinella, E. Bianchi, M. Finotto, G. Cantoni, D. M. Eckert, V. M. Supekar, C. Bruckmann, A. Carfi, and A. Pessi, Structural characterization of the fusion-active complex of severe acute respiratory syndrome (SARS) coronavirus, Proc. Natl. Acad. Sci. USA 101, 8709-8714 (2004).

16. Y. Xu, Z. Bai, L. Qin, X. Li, G. Gao, and Z. Rao, Crystallization and preliminary crystallographic analysis of the fusion core of the spike protein of the murine coronavirus mouse hepatitis virus (MHV), Acta Crystallogr. D 60, 2013-2015 (2004). 